\title{
INNER PRODUCTS IN NORMED LINEAR SPACES
}

\author{
ROBERT C. JAMES
}

Let $T$ be any normed linear space $\left[1\right.$, p. 53]. ${ }^{1}$ Then an inner prod$u c t$ is defined in $T$ if to each pair of elements $x$ and $y$ there is associated a real number $(x, y)$ in such a way that $(x, y)=(y, x),\|x\|^{2}=(x, x)$, $(x, y+z)=(x, y)+(x, z)$, and $(t x, y)=t(x, y)$ for all real numbers $t$ and elements $x$ and $y$. An inner product can be defined in $T$ if and only if any two-dimensional subspace is equivalent to Cartesian space [5]. A complete separable normed linear space which has an inner product and is not finite-dimensional is equivalent to (real) Hilbert space, ${ }^{2}$ while every finite-dimensional subspace is equivalent to Euclidean space of that dimension. Any complete normed linear space $T$ which has an inner product is characterized by its (finite or transfinite) cardinal "dimension-number" $n$. It is equivalent to the space of all sets $x=\left(x_{1}, x_{2}, \cdots\right)$ of $n$ real numbers satisfying $\sum_{1} x_{i}^{2}<+\infty$, where $\|x\|=\left(\sum_{i} x_{i}^{2}\right)^{1 / 2}[7$, Theorem 32]. Various necessary and suffcient conditions for the existence of an inner product in normed linear spaces of two or more dimensions are known. Two such conditions are that $\|x+y\|^{2}+\|x-y\|^{2}=2\left[\|x\|^{2}+\|y\|^{2}\right]$ for all $x$ and $y$, and that $\lim _{n \rightarrow \infty}\|x+n y\|-\|n x+y\|=0$ whenever $\|x\|=\|y\|$ ([5] and [4, Theorem 6.3]). A characterization of inner product spaces of three or more dimensions is that there exist a projection of unit norm on each twodimensional subspace [6, Theorem 3$]$. Other characterizations valid for three or more dimensions will be given here, expressed by means of orthogonality, hyperplanes, and linear functionals.

A hyperplane of a normed linear space is any closed maximal linear subset $M$, or any translation $x+M$ of $M$. A hyperplane is a supporting hyperplane of a convex body $S$ if its distance from $S$ is zero and it does not contain an interior point of $S$; it is tangent to $S$ at $x$ if it is the only supporting hyperplane of $S$ containing $x[8, \mathrm{pp} .70-74]$. It will be said that an element $x_{0}$ of $T$ is orthogonal to $y\left(x_{0} \perp y\right)$ if and only if $\left\|x_{0}+k y\right\| \geqq\left\|x_{0}\right\|$ for all $k$, which is equivalent to requiring the existence of a nonzero linear functional $f$ such that $f\left(x_{0}\right)=\|f\|\left\|x_{0}\right\|$ and $f(y)=0$, or that $x_{0}+y$ belong to a supporting hyperplane of the sphere

Presented to the Society, November 2, 1946; received by the editors December 31, 1946.

1 Numbers in brackets refer to the references at the end of the paper.

2 "Equivalent" meaning isometric under a linear transformation [1, p. 180]. The equivalence to (real) Hilbert space follows by reasoning similar to that of $[10, \mathrm{pp}$. 3-16]. 
$\|x\| \leqq\left\|x_{0}\right\|$ at the point $x_{0}[4$, Theorem 2.1 and $\$ 5]$. In a space with an inner product, $x \perp y$ if and only if $(x, y)=0$.

Orthogonality is said to be additive on the right if and only if $z \perp x$ and $z \perp y$ imply $z \perp x+y$. Clearly $x \perp x$ implies $x=0$, while $x \perp y$ implies $a x \perp b y$ for any numbers $a$ and $b$. Every element is orthogonal to at least one hyperplane through the origin, this hyperplane being unique for any given element if and only if: (1) For any $x(\neq 0)$ and $y$ there is a unique number $a$ with $x \perp a x+y$; (2) The unit sphere $\|x\| \leqq 1$ of $T$ has a tangent hyperplane at each point; (3) The norm is Gateaux differentiable; or (4) Orthogonality is additive on the right [4, Theorems 4.2, 5.1].

Orthogonality is said to be additive on the left if and only if $x \perp z$ and $y \perp z$ imply $x+y \perp z$. Orthogonality is not symmetric in general, and there does not necessarily exist a hyperplane orthogonal to a given element (Theorems 1 and 5). Additivity on the left does not imply strict convexity, ${ }^{3}$ nor conversely, but a normed linear space is strictly convex if and only if: (1) For any $x(\neq 0)$ and $y$ there is a unique number $a$ with $a x+y \perp x$; or (2) No supporting hyperplane has more than one point of contact [4, Theorems 4.3, 5.2].

Birkhoff has shown that an inner product can be defined in a normed linear space of three or more dimensions if orthogonality is symmetric and unique. ${ }^{4}$ An equivalent condition is that $N_{+}(x ; y)=0$ whenever $N_{+}(y ; x)=0$, where $N_{+}(x ; y)=\lim _{h \rightarrow+0}[\|x+h y\|-\|x\|] / h$ exists because of the convexity of the function $f(h)=\|x+h y\|[4$, Theorem 6.2]. It is possible to show by a purely geometric argument that in a space of three or more dimensions orthogonality must be unique if it is symmetric, but this follows more easily from known facts about projections in normed linear spaces:

THEOREM 1. Orthogonality is symmetric in a normed linear space $T$ of three or more dimensions if and only if an inner product can be defined in $T$.

Proof. Let $x_{1}$ and $x_{2}$ be any two elements of a three-dimensional subspace $T_{0}$ of $T$. Then there is an element $y \in T_{0}$ orthogonal to the linear hull $H_{0}$ of $x_{1}$ and $x_{2}$ [4, Theorem 7.1]. If orthogonality is symmetric, then $H_{0} \perp y$. Hence if a projection of $T_{0}$ on $H_{0}$ is defined by $z=P(z)+a_{z} y$, where $P(z) \in H_{0}$, then $\|P(z)\| \leqq\|z\|$ for all $z$ and $\|P\|=1$. But it is known that an inner product can be defined in a normed

\footnotetext{
${ }^{3}$ A normed linear space is strictly convex if $\|x+y\|=\|x\|+\|y\|$ and $y \neq 0$ imply $x=t y$ for some $t$.

4 See [2]. With symmetry, uniqueness means the uniqueness for any $x(\neq 0)$ and $y$ of the number $a$ for which $x \perp a x+y$.
} 
linear space of three or more dimensions if there is a projection of norm one on any given closed linear subspace [6, Theorem 3]. Thus an inner product can be defined in any three-dimensional subspace of $T$ and hence in $T$ itself [5].

For elements $x$ and $y$ of a normed linear space, $x \perp y$ if and only if there is a nonzero linear functional $f$ such that $f(x)=\|f\|\|x\|$ and $f(y)=0$, while $a x+y \perp x$ if and only if $\|k x+y\|$ is minimum for $k=a$ $[4$, Theorems $2.1,2.3]$. Also, the set $H$ of all $z$ satisfying $f(z)=\|f\|$ is a supporting hyperplane of the unit sphere at $x$ if $f(x)=\|f\|$ and $\|x\|=1$, while any supporting hyperplane can be defined by such an equation (see Mazur [8, p. 71]). Also, $H$ is said to be parallel to an element $y$ if and only if $f(y)=0$ (that is, the line $\{k y\}$ does not intersect $H$ ). Interpretations of Theorem 1 by means of linear functionals and hyperplanes therefore give the following necessary and sufficient conditions for the existence of an inner product in a normed linear space of three or more dimensions:

(1) For any elements $x$ and $y$, the existence of a nonzero linear functional $f$ with $f(x)=\|f\|\|x\|$ and $f(y)=0$ implies the existence of a nonzero linear functional $g$ with $g(y)=\|g\|\|y\|$ and $g(x)=0$.

(2) For any elements $x$ and $y,\|k x+y\|$ is minimum when $k=-f(y) / f(x)$ if $f$ is a linear functional with $f(x)=\|f\|\|x\|$.

(3) The existence of a supporting hyperplane of the unit sphere at $x$ parallel to $y(\|x\|=\|y\|=1)$ implies the existence of a supporting hyperplane at $y$ parallel to $x$.

There are infinitely many different normed linear spaces of two dimensions in which orthogonality is not symmetric [2, Theorem 4]. If an isomorphism $a x+b y \leftrightarrow(a, b)$ is set up between the Cartesian plane and a two-dimensional normed linear space containing $x$ and $y$ $(\|x\|=\|y\|=1)$ and if $C$ is the "unit pseudo-circle" of all points $(a, b)$ for which $\|a x+b y\|=1$, then orthogonality is symmetric in $T$ if and only if the line through the origin parallel to any supporting line of $C$ at any point $p$ cuts $C$ in a point at which there is a supporting line parallel to the line from $p$ to the origin. Let $B_{r}(r \geqq 1)$ be the normed linear space of pairs $\left(x_{1}, x_{2}\right)=x$ of real numbers, where $\|x\| r=\left(\left|x_{1}\right| r+\left|x_{2}\right|^{r}\right)$ if $x_{1}$ and $x_{2}$ are of the same sign, and $\|x\|^{s}$ $=\left(\left|x_{1}\right|^{s}+\left|x_{2}\right|^{s}\right)$ otherwise, where $s=r /(r-1)$. It can easily be verified that orthogonality is symmetric in $B_{r}$ for $r \geqq 1$, and that it is unique except in the limiting case $r=1$. Thus orthogonality can be symmetric and not unique in a two-dimensional space.

Theorem 2. An inner product can be defined in a normed linear space of three or more dimensions if and only if orthogonality is additive on the left. 
Proof. Let $T$ be a normed linear space of three or more dimensions, and $x_{1}$ and $x_{2}$ be any two elements. Then there are hyperplanes $H_{1}$ and $H_{2}$ with $x_{1} \perp H_{1}$ and $x_{2} \perp H_{2}$. Let $M=H_{1} \cap H_{2}$. If orthogonality is additive on the left, then $a x_{1}+b x_{2} \perp M$ for all $a$ and $b$, and any element $z$ has a unique representation in the form $z=P(z)+y$, where $y \in M$ and $P(z)=a x_{1}+b x_{2}$. Also, $\|z\| \geqq\|P(z)\|$ for all $z$, and $\|P\|=1$. Since there is a projection of norm one on any given two-dimensional linear subspace of $T$, it follows as for Theorem 1 that an inner product can be defined in $T$ [ 6 , Theorem 3$]$.

The conclusion of the above theorem is not valid without the assumption that the space be of more than two dimensions, since it is clear that for a two-dimensional normed linear space orthogonality is additive on the left if and only if for any $x(\neq 0)$ there is a unique nonzero element orthogonal to $x$. It therefore follows that orthogonality is additive on the left in a two-dimensional normed linear space if and only if the space is strictly convex [4, Theorem 4.3].

If $L$ is a closed linear set in a Banach space $B$, then the normal projection of $x$ on $L$ is said to be the element $u$ for which $x-u \perp L$, or for which $\|x-u\|$ is the distance from $x$ to $L$. If $L$ is finite-dimensional, or if the unit sphere of $B$ is weakly compact, then normal projection is defined for all $x$ and $L$ [4, Theorem 7.2]. It was shown by Fortet $[3, \mathrm{p} .45]$ that if orthogonality is symmetric in a uniformly convex Banach space, then normal projection is a continuous linear operation and the set $H$ of points $y$ with $y \perp x$ is linear and closed. However, it follows from the above theorems that $H$ is linear for all $x$ only if an inner product can be defined in the space $R$ and that the existence of an inner product follows from symmetry of orthogonality. Also, $x \perp L$ if and only if there is a linear functional $f$ with $f(x)=\|f\|\|x\|$ and $f(L)=0$ $[4$, Theorem 2.1]. The following characterizations of inner product spaces of three or more dimensions are therefore direct consequences of Theorem 2.

(4) The existence of a linear functional $F$ with $F(x+y)=\|F\|\|x+y\|$ and $F(z)=0$ whenever $x, y$, and $z$ are such that there are linear functionals $f$ and $g$ with $f(x)=\|f\|\|x\|, g(y)=\|g\|\|y\|$, and $f(z)=g(z)=0$.

(5) That normal projection be a linear operation.

If a complete normed linear space has an inner product, then any linear functionals $f$ and $g$ can be written in the form $f(u)=(x, u)$ and $g(u)=(y, u)$, for some elements $x$ and $y$ [7, Theorem 11]. Then $F$ of (4) can be taken as $f+g$. For any linear functional $G=A f+B g$, there are then numbers $a$ and $b$ such that $G(a x+b y)=\|G\|\|a x+b y\|$. This condition is also sufficient for an inner product: 
THEOREM 3. An inner product can be defined in a normed linear space $T$ of three or more dimensions if and only if it follows from $f(x)=\|f\|\|x\|$ and $g(y)=\|g\|\|y\|$ for linear fundtionals $f$ and $g$ and elements $x$ and $y$ of $T$ that there are numbers $a$ and $b$ such that $f(a x+b y)+g(a x+b y)$ $=\|f+g\|\|a x+b y\|$ and $a x+b y \neq 0$.

Proof. First note that if for some $x$ there are two nonzero linear functionals $F$ and $G$ with $F(x)=\|F\|\|x\|$ and $G(x)=\|G\|\|x\|$, then the assumption of the theorem would imply that $|h(x)|=\|h\|\|x\|$ if $h=\|G\| F-\|F\| G$. But this is clearly impossible unless $h \equiv 0$, or $\|G\| F=\|F\| G$. Thus two independent linear functionals cannot take on their maximum in the unit sphere $\|x\| \leqq 1$ at the same point, which is known to imply that the unit sphere has a tangent hyperplane at each point [4, Theorem 5.1]. Now suppose that $x \perp z$ and $y \perp z$, and let $T_{0}$ be the linear hull of $x, y$, and $z$. There are then two linear functionals $f$ and $g$ with $f(x)=\|f\|\|x\|, g(y)=\|g\|\|y\|$, and $f(z)=g(z)=0$ [4, Theorem 2.1]. If $x$ and $y$ are not linearly independent, then $x+y \perp z$. Let $x$ and $y$ be linearly independent and suppose that for $u=x+y$ there are no numbers $A$ and $B$ satisfying $|A f(u)+B g(u)|$ $=\|A f+B g\|\|u\|$. Let $C$ be the curve of all elements $a x+b y$ with $\|a x+b y\|=1$. Then there are elements $x^{\prime}$ and $y^{\prime}$ on either side of $(x+y) /\|x+y\|$ and in $C$ for which there are linear functionals $f^{\prime}=A_{1} f+B_{1} g$ and $g^{\prime}=A_{2} f+B_{2} g$ with $f^{\prime}\left(x^{\prime}\right)=\left\|f^{\prime}\right\|\left\|x^{\prime}\right\|$ and $g^{\prime}\left(y^{\prime}\right)$ $=\left\|g^{\prime}\right\|\left\|y^{\prime}\right\|$, but such that none of the linear functionals $A f^{\prime}+B g^{\prime}$ satisfy $\left|A f^{\prime}\left[r x^{\prime}+(1-r) y^{\prime}\right]+B g^{\prime}\left[r x^{\prime}+(1-r) y^{\prime}\right]\right|=\left\|A f^{\prime}+B g^{\prime}\right\| \| r x^{\prime}+$ $(1-r) y^{\prime} \|$ for any $r$ with $0<r<1$. For each such $r$, there is a number $\alpha_{r}$ for which $\left[r x^{\prime}+(1-r) y^{\prime}+\alpha_{r} z\right] /\left\|r x^{\prime}+(1-r) y^{\prime}+\alpha_{r} z\right\|=v \perp z \quad[4$, Theorem 2.3]. If $h$ is a linear functional defined in $T_{0}$ for which $h(v)=\|h\|\|v\|$ and $h(z)=0$, and if $A_{r}$ and $B_{r}$ are such that $A_{r} f^{\prime}\left(z_{0}\right)$ $+B_{r} g^{\prime}\left(z_{0}\right)=0$ for some $z_{0} \in T_{0}$ for which $h=0$ but not both $f^{\prime}$ and $g^{\prime}$ are zero, then $h$ and $A_{r} f^{\prime}+B_{r} g^{\prime}$ are both zero at $z_{0}$ and $z$ and hence are multiples of each other on $T_{0}$. Then if $a_{r}$ and $b_{r}$ are chosen by the assumptions of the theorem so that $\left\|a_{r} x^{\prime}+b_{r} y^{\prime}\right\|=1$ and $\mid A_{r} f^{\prime}\left(a_{r} x^{\prime}\right.$ $\left.+b_{r} y^{\prime}\right)+B_{r} g^{\prime}\left(a_{r} x^{\prime}+b_{r} y^{\prime}\right) \mid=\left\|A_{r} f^{\prime}+B_{r} g^{\prime}\right\|$, it follows that $h$ is a multiple of $A_{r} f^{\prime}+B_{r} g^{\prime}$ and that $h\left(a_{r} x^{\prime}+b_{r} y^{\prime}\right) \mid=\|h\|\left\|a_{r} x^{\prime}+b_{r} y^{\prime}\right\|$. Thus the unit sphere $S$ contains the straight lines $l_{r}$ between $a_{r} x^{\prime}+b_{r} y^{\prime}$ and $v$, since the unit sphere is convex and the tangent hyperplane defined by $h(x)=\|h\|$ contains $a_{r} x^{\prime}+b_{r} y^{\prime}$ and $v$. This tangent hyperplane at $v$ then contains this line, but does not contain a point of $C$ between $x^{\prime}$ and $y^{\prime}$. But there are also tangent hyperplanes at $x^{\prime}$ and $y^{\prime}$ parallel to $z$, while $a_{r} x^{\prime}+b_{r} y^{\prime}$ is by assumption not of the form $\left[r x^{\prime}+(1-r) y^{\prime}\right] / \| r x^{\prime}$ $+(1-r) y^{\prime} \|$ for any $r$ satisfying $0<r<1$. This implies that the tan- 
gent hyperplane at $v$ contains either $x^{\prime}$ or $y^{\prime}$ and is coincident with the tangent hyperplane at $x^{\prime}$ or $y^{\prime}$, respectively. Letting $r$ vary from 0 to 1 , it now follows from the convexity of $S$ that the tangent hyperplanes at $x^{\prime}$ and at $y^{\prime}$ have a common point of contact and must therefore coincide, since $S$ has a tangent hyperplane at each point. This tangent hyperplane then contains the line from $x^{\prime}$ to $y^{\prime}$, and $f^{\prime}(x+y)=\left\|f^{\prime}\right\|\|x+y\|$, contrary to assumption. Hence there are numbers $A$ and $B$ with $\mid A f(x+y)$ $+B g(x+y) \mid=\|A f+B g\|\|x+y\|$. Since $A f(z)+B g(z)=0$, this implies that $x+y \perp z$ and that orthogonality is additive on the left. It now follows from Theorem 2 that an inner product can be defined in $T$.

For any element $x$ of a normed linear space there is always a hyperplane $H$ through the origin with $x \perp H$. However, for no hyperplane $H$ of the space ${ }^{5} C$ of continuous functions is there an element $f \in C$ with $H \perp f$. This follows from the fact that $g \perp f$ if and only if $\min _{A} g f \leqq 0 \leqq \max _{A} g f$, where $A$ is the set of all $t$ with $|g(t)|=\|g\|$ $[4, \S 4]$. If $T$ is one of the spaces ${ }^{5}(s),(m),(c)$, or $l(p)(p \geqq 1)$, then clearly $H \perp x$ for an infinite number of different hyperplanes $H$ and elements $x$. If a normed linear space is strictly convex, then for no element $x$ is there more than one hyperplane $H$ with $H \perp x$, while no hyperplane is orthogonal to more than one element if the norm of $T$ is differentiable $[4$, Theorems $4.2,4.3]$. This difference is the reason for the lack of similarity between the proofs of the following theorems.

TheOREM 4. An inner product can be defined in a normed linear space of three or more dimensions if and only if each hyperplane through the origin is orthogonal to at least one element.

PRoof. Let $x_{1}$ and $x_{2}$ be any two elements of a normed linear space $T$ of three or more dimensions, and let $P_{0}$ be the linear hull of $x_{1}$ and $x_{2}$. By well-ordering the set of all linear subspaces $M$ of $T$ for which $P_{0} \perp M$, it follows that there is a linear subspace $\bar{M}$ of $T$ such that $P_{0} \perp \bar{M}$ and $\bar{M}$ is not contained properly in any other such linear subspace. Then it is clear that $\bar{M}$ is closed. Hence if the linear hull $H$ of $P_{0}$ and $\bar{M}$ were not $T$, there would be a hyperplane through the origin which contains $P_{0}$ and $\bar{M}$. If every hyperplane through the origin is orthogonal to some element, then there would be an element $x$ such that $H \perp x$. But if $y=x_{p}+x_{m}+k x$, where $x_{p} \in P_{0}$ and $x_{m} \in \bar{M}$, then $\|y\| \geqq\left\|x_{p}+x_{m}\right\| \geqq\left\|x_{p}\right\|$, since $\left(x_{p}+x_{m}\right) \perp x$ and $x_{p} \perp x_{m}$. Thus $P_{0}$ would be orthogonal to the linear hull of $\bar{M}$ and $x$. Hence the linear hull of $P_{0}$ and $\bar{M}$ must be $T$. A projection $P(z)$ of $T$ on $P_{0}$ can now be defined by $z=P(z)+z_{m}$, where $P(z) \in P_{0}$ and $z_{m} \in \bar{M}$. Since $\|P\|=1$,

5 The notation is that of Banach [1, pp. 10-12]. 
it follows that there is a projection of unit norm on any given twodimensional linear subspace of $T$ and hence (as in the proof of Theorem 1) that an inner product can be defined in $T$.

TheOREM 5. An inner product can be defined in a normed linear space $T$ of three or more dimensions if and only if for any $x \in T$ there is a hyperplane $H$ through the origin with $H \perp x$.

Proof. Suppose $x, y$, and $z$ are any three elements of $T$ with $x \perp z$ and $y \perp z$. If $T$ is strictly convex, then for any $u$ and $v$ of $T$ there is a unique $a$ such that $a u+v \perp u[4$, Theorem 4.3]. Hence if $H$ is a hyperplane through the origin with $H \perp z$, and if $T$ is strictly convex, then $x \in H$ and $y \in H$. Thus $x+y \in H$ and $x+y \perp z$, orthogonality is additive on the left, and an inner product can be defined in $T$. Now suppose $T$ is not strictly convex. Then there are elements $x$ and $y$ and a linear functional $f$ with $f(x)=f(y)=\|f\|$ and $\|x\|=\|y\|=1$ [9, Theorem 6]. Let $z$ be any other element of unit norm not in the linear set generated by $x$ and $y$ and let $S_{0}$ be the unit sphere of the space $T_{0}$ generated by $x, y$, and $z$. Let $P_{0}$ be the set of all points $u \in S_{0}$ for which $\|u\|=1$ and $f(u)=\|f\|$. Then $P_{0}$ contains the line from $x$ to $y$, and is itself either a straight line segment or a section of a plane. Let $L_{0}$ be the hyperplane of $T_{0}$ with $P_{0} \perp L_{0}$, where $L_{0}$ contains all points at which $f$ is zero. Then for any $v$ and each number $a$ there is a hyperplane $H_{a}$ of $T_{0}$ with $H_{a} \perp v+a x$. As $a \rightarrow+0$ (or as $a \rightarrow-0$ ), the planes $H_{a}$ will have at least one limit $H_{+}$(or $H_{-}$) in the sense that there exist sequences $\left\{a_{i}\right\}$ and $\left\{b_{i}\right\}$, with $a_{i} \rightarrow+0$ and $b_{i} \rightarrow-0$, $\lim _{a_{i} \rightarrow+0} \rho\left(w, H_{a_{i}}\right)=0$ and $\lim _{b_{i} \rightarrow-0} \rho\left(w, H_{b_{i}}\right)=0$, if $w$ is any fixed element of $H_{+}$or $H_{-}$, respectively. Since at each point of unit norm in $H_{a}$ there is a supporting plane of $S_{0}$ parallel to $v+a x$, it follows that if $v \in L_{0}$ then neither $H_{+}$nor $H_{-}$crosses $P_{0}$, and $P_{0}$ consists of those and only those points of the surface of $S_{0}$ in a region containing $x$ and bounded by $H_{+}, H_{-}$, and the two supporting lines of $P_{0}$ parallel to $v$. But this is possible for arbitrary $v \in L_{0}$ only if $P_{0}$ is a point.

Theorems 3-5 can be given direct interpretations by means of supporting hyperplanes of the unit sphere $S$, as was done for Theorem 1 to get (3). The first of these interpretations can be changed somewhat to give the following nontrivial consequence of Theorem 3 .

THEOREM 6. An inner product can be defined in a Banach space if every supporting hyperplane of the unit sphere $S$ has a point of contact and the existence of supporting hyperplanes $H_{1}$ and $H_{2}$ at points $x$ and $y$ of $S$ imply that any supporting hyperplane $H_{3}$ of $S$ satisfying $H_{1} \cap H_{2}$ $\cap H_{3}=0$ have a point of contact which is in the linear hull of $x$ and $y$. 
Proof. First suppose that there is an element $x$ and nonzero linear functionals $f_{1}$ and $f_{2}$ such that $\|x\|=1, f_{1}(x)=\left\|f_{1}\right\|$, and $f_{2}(x)=\left\|f_{2}\right\|$. Then $x$ is in both of the supporting hyperplanes $H_{1}$ and $H_{2}$ of $S$, where $H_{1}$ and $H_{2}$ are defined by $f_{1}(z)=\left\|f_{1}\right\|$ and $f_{2}(z)=\left\|f_{2}\right\|$. If $L$ is the set of points at which $f_{1}=f_{2}=0$, then $H_{1} \cap H_{2}=x+L$. If $f_{1}$ and $f_{2}$ are linearly independent, then the linear hull of $x$ and $L$ is not the whole space and there is a nonzero linear functional $f_{3}$ which is zero on $x$ and $L$. Let $H_{3}$ be defined by $z \in H_{3}$ if and only if $f_{3}(z)=\left\|f_{3}\right\|$. Then clearly $H_{1} \cap H_{2} \cap H_{3}=0$. But the second hypothesis of the theorem would imply that $x \in H_{3}$, or $f_{3}(x)=\left\|f_{3}\right\|$, which contradicts $f_{8}(x)=0$. Therefore $f_{1}$ and $f_{2}$ are linearly dependent.

Now suppose that $\|x\|=\|y\|=1, f_{1}(x)=\left\|f_{1}\right\|$, and $f_{2}(y)=\left\|f_{2}\right\|$. If $f_{3}=f_{1}+f_{2}$, and $H_{1}, H_{2}, H_{3}$ are defined by $f_{i}(z)=\left\|f_{i}\right\|(i=1,2,3)$, then $x \in H_{1}$ and $y \in H_{2}$, If $H_{1} \cap H_{2} \cap H_{3} \neq 0$, then there exists an element $w$ such that $f_{1}(w)=\left\|f_{1}\right\|, f_{2}(w)=\left\|f_{2}\right\|$, and $f_{1}(w)+f_{2}(w)=\left\|f_{1}+f_{2}\right\|$. Thus $\left\|f_{1}+f_{2}\right\|=\left\|f_{1}\right\|+\left\|f_{2}\right\|$. Since every linear functional in $T$ takes on its maximum in the unit sphere, $H_{3}$ contains a point $z$ of norm 1 . Then $f_{1}(z)+f_{2}(z)=\left\|f_{1}+f_{2}\right\|=\left\|f_{1}\right\|+\left\|f_{2}\right\|$. Therefore $f_{1}(z)=\left\|f_{1}\right\|$ and $f_{2}(z)$ $=\left\|f_{2}\right\|$. Hence $f_{1}$ and $f_{2}$ must be linearly dependent, and $f_{1}(x)+f_{2}(x)$ $=\left\|f_{1}+f_{2}\right\|$. If $H_{1} \cap H_{2} \cap H_{3}=0$, then $H_{3}$ has a point of contact $a x$ $+b y(\|a x+b y\|=1)$ and $f_{3}(a x+b y)=\left\|f_{3}\right\|\|a x+b y\|$. Thus it follows from Theorem 3 that an inner product can be defined.

\section{REFERENCES}

1. S. Banach, Theorie des operations linéaires, Warsaw, 1932.

2. G. Birkhoff, Orthogonality in linear metric spaces, Duke Math. J. vol. 1 (1935) pp. 169-172.

3. R. Fortet, Remarques sur les espaces uniformement convexes, Bull. Soc. Math. France vol. 69 (1941) pp. 23-46.

4. R. C. James, Orthogonality and linear functionals in normed linear spaces, Trans. Amer. Math. Soc. vol. 61 (1947) pp. 265-292.

5. P. Jordan and J. von Neumann, On inner products in linear, metric spaces, Ann. of Math. vol. 36 (1935) pp. 719-723.

6. S. Kakutani, Some characterizations of Euclidean space, Jap. J. Math. vol. 16 (1939) pp. 93-97.

7. H. Löwig, Komplexe Euklidische Räume von beliebiger endlicher oder transfiniter Dimensionszahl, Acta Litterarum ac Scientiarum vol. 7 (1934-1935) pp. 1-33.

8. S. Mazur, Über convexe Mengen in linearen normierten Raumen, Studia Mathematica vol. 4 (1933) pp. 70-84.

9. V. Smulian, On some geometrical properties of the unit sphere in the space of the type (B), Rec. Math. (Mat. Sbornik) N.S. vol. 48 (1938) pp. 90-94.

10. M. H. Stone, Linear transformations in Hilbert space, Amer. Math. Soc. Colloquium Publications, vol. 15, New York, 1932.

HARVARD UNIVERSITY 